\title{
Review of: "The C-terminal domain of Hsp70 is responsible for paralog-specific regulation of ribonucleotide reductase"
}

\author{
Tawanda Zininga \\ 1 University of Stellenbosch
}

Potential competing interests: The author(s) declared that no potential competing interests exist.

Dear Editor

I have reviewed the manuscript submitted by the authors titled: the C-terminal domain of Hsp70 is responsible for paralogy specific regulation of ribonucleotide reductase. The manuscript is a detailed account of analysing the potential role of the c-terminal domain of yeast Hsp70 (Ssa1-4) paralogs in modulating chaperone function specificity. The cterminal domain of the Ssa2 and Ssa4 were swapped and reported to be responsible for dictating chaperone function in a complementation assay. This suggest that the C-terminal domain forms unique interaction with a potential substrate, ribonucleotide reductase and co-chaperones to facilitate the chaperone functions. The introduction and the discussion are focused and sufficient for this MS. However, a few minor errors were picked in the methods and results section that needs to be addressed before acceptance of the manuscript. In short, the experimental design and statistical analysis of the data should be re-looked at, as there seems to confusion on the definition of replicates vs repeats as well as the use of standard deviation vs standard errors.

Please find below my detailed report on errors that need to be corrected to improve the quality of the manuscript. Line 126, the 2 panels for cell viability and IC50 bar graphs should be both mentioned and explained how each was constructed.

Line 126, The figure 1 (A-D) bar graphs $y$-axis scale and the font for $E$ should be uniform with the rest of the figures. Line 127, The cell damaging agents are not all represented in figure 1A. This should be corrected to represent the damaging agent in $A, B, C$ and $D$, respectively.

Line 131, The DNA damage response-regulated transcription should have been confirmed using another DNA damaging agent to validate the findings from hydroxyurea.

Line 142, the ratios below the blots are not explained how they were determined.

Line 144, the presence of a doublet on the ssa1 and ssa2 blot should be explained as well as the lack of distinct lanes in the PGK1 control.

Line 146, insert 'expression' after Rnr4 induced.

Line 146, The blot for Fig $2 \mathrm{C}$ is inconsistent with the reported findings as it seems as if there was less loaded protein in Ssa3 lanes which does not suggest lower induction levels.

Line 153, the panel with RNR4 RT-qPCR y-axis are title is missing 
Line 156, the findings for Rnr2 stability are not consistent with the blot provided in figure 3B.

Line 157, There is reference to figure $3 \mathrm{C}$ which does not exist in the manuscript.

Line 165, the $y$-axis scale should be uniform for A-C, in panel A the letter BD is missing on panels B and C.

Line 177, the Ydj1-Rnr4 interaction on figure 5C is not consistent with description. This should be explained in the context of absence of $\mathrm{HU}$ as $\mathrm{HU}$ treated cells suggest interaction. For example, the IP blot ratio for Ssa2 untreated is represented as 144 versus the ratio of Ssa3 treated. Figure 5D is not mentioned and described in the main text.

Line 182, the text on the alignment figure $6 \mathrm{~A}$ is blurry, increase resolution by focusing on the swapped domains.

Line 187, The differences between Ssa2 and Ssa4 resistance should be semi-quantified to make comparison with Ssa24 clear.

Line 193, the font size for figure 6D should be consistent with the other figures in the manuscript.

Line 331, mention the city and country for the manufactures of reagents and equipment used and do this throughout the MS.

Lines 662, 676, How were the statistical validations conducted, on replicates or repeats as defined in Vaux et al., 2012 EMBO reports vol13 no. 4, 291-296.

Line 679, delete the repeated words 'transformed with'.

Line 694, how was $\mathrm{n}=3$ defined as repeats or replicates? 